\title{
Propranolol en el Tratamiento de los Hemangiomas de la Infancia
}

\author{
DANIELA KRÄMER H. ${ }^{1}$, PAULA MUÑOZ M. ${ }^{1}$, PATRICIA ALFARO C. ${ }^{2}$, \\ LIGIA ARANÍBAR D. ${ }^{1}$, LUIS CÁRDENAS M. ${ }^{3}$, CARMEN GLORIA MOROVIC I. ${ }^{4}$, \\ CLAUDIA VIDAL T. ${ }^{4}$, CAROLINA WHITTLE P. ${ }^{5}$ \\ 1. Dermatólogo. Unidad de Dermatología, Hospital Luis Calvo Mackenna. \\ 2. Médico en Etapa de Destinación y Formación. \\ 3. Cardiólogo-Pediatra. Servicio Cardiología, Hospital Luis Calvo Mackenna. \\ 4. Cirujano Plástico Pediátrico. Servicio de Cirugía Hospital Luis Calvo Mackenna. \\ 5. Radióloga, Servicio de Ecotomografía, Departamento de Imágenes. Clínica Alemana.
}

\begin{abstract}
Propranolol in the Treatment of Infantile Hemangioma

Infantile Hemangiomas $(\mathrm{IH})$ are common vascular tumors in children. Only $10-15 \%$ should be treated by any vital, functional or aesthetic complications. Oral corticosteroids are the treatment of choice. However, in the last two years an excellent response to treatment of hemangiomas with oral Propranolol has been reported. Objective: evaluate this new therapy in a limited number of chilean patients with $\mathrm{IH}$ with an indication of systemic treatment and present this experience. Patients and Methods: We present 30 cases of IH treated at our institution with oral Propranolol, using a dose of $2 \mathrm{mg} / \mathrm{kg} /$ day divided in 2 daily doses. Results: 77\% of patients were female, $40 \%$ hemangiomas had segmental distribution, $90 \%$ were solitary, $67 \%$ were located in the head and neck and 23\% were ulcerated. The treatment started at an average of 119 days of life and lasted for an average of 7 months. In all our patients the use of Propranolol accelerated the onset of involution of the hemangiomas and decreased color, brightness and growth too. $43 \%$ of the patients had irritability and sleep disorders. No significant adverse effects were reported in any of our patients. Conclusion: Propranolol treatment was highly effective and safe in our series of patients and we propose, taking into account published reports based on this data, it should become the treatment of choice for IH.
\end{abstract}

(Key words: Propranolol, Treatment, Infantile Hemangiomas).

Rev Chil Pediatr 2010; 81 (6): 523-530

\section{RESUMEN}

Los Hemangiomas de la Infancia (HI) son tumores vasculares frecuentes en la edad pediátrica. Sólo un 10$15 \%$ debe ser tratado por eventuales consecuencias vitales, funcionales o estéticas. Los corticoides orales son el tratamiento de elección actual. Sin embargo, en los últimos 2 años se ha reportado la excelente respuesta de los hemangiomas al tratamiento con Propranolol oral. Objetivo: Evaluar esta nueva terapéutica en un número limitado de pacientes Chilenos con hemangiomas de la infancia con indicación de tratamiento sistémico y

Trabajo recibido el 04 de agosto de 2010, devuelto para corregir el 28 de septiembre de 2010, segunda versión el 01 de octubre de 2010, aceptado para publicación el 04 de octubre de 2010.

Correspondencia a:

Daniela Krämer $\mathrm{H}$.

E-mail: danielakramer.chile@gmail.com 
presentar esta experiencia. Pacientes y Método: Presentamos 30 casos de HI tratados en nuestra institución con Propranolol oral, usando una dosis de $2 \mathrm{mg} / \mathrm{kg} /$ día en 2 tomas diarias. Resultados: Un 77\% de los pacientes eran de sexo femenino. El 40\% de los hemangiomas eran segmentarios, $90 \%$ eran únicos, $67 \%$ estaban ubicados en cabeza y cuello y $23 \%$ estaban ulcerados. El tratamiento se inició en promedio a los 119 días de vida y duró en promedio 7 meses. En la totalidad de nuestros pacientes el uso de Propranolol aceleró el inicio de la involución del hemangioma y disminuyó el color, brillo y crecimiento. El $43 \%$ de los pacientes presentó irritabilidad y trastornos del sueño. No se presentaron efectos adversos importantes en ninguno de nuestros pacientes. Conclusión: El tratamiento con Propranolol fue altamente efectivo y seguro en nuestra serie de pacientes y proponemos, tomando en cuenta los reportes publicados al respecto, que debiera ser el tratamiento de elección para los hemangiomas de la infancia

(Palabras clave: Propranolol, tratamiento, hemangiomas de la infancia).

Rev Chil Pediatr 2010; 81 (6): 523-530

\section{Introducción}

Los Hemangiomas de la Infancia (HI) son tumores vasculares, de naturaleza benigna, con características biológicas muy específicas que los diferencian de otros tumores vasculares ${ }^{1}$.

Son los tumores benignos más frecuentes de la infancia. Se presentan en un $5-10 \%$ de los niños menores de un año ${ }^{1-3}$, tienen un claro predominio en el sexo femenino (relación 4:1) y en los recién nacidos prematuros en los cuales la incidencia se relaciona en forma inversa con el peso de nacimiento ${ }^{1,2,4}$.

Los HI tienen un comportamiento biológico único. Se caracterizan por ser lesiones inaparentes o escasamente desarrolladas al momento de nacer, crecer a una velocidad variable durante los primeros 12 a 18 meses de vida y luego involucionar espontáneamente por varios años ${ }^{1,5}$. La regresión completa se produce en un $60 \%$ de los niños a los 4 años y en un $76 \%$ a los 7 o más años ${ }^{6}$. Un $20-40 \%$ de los pacientes queda con alguna secuela residual, especialmente las lesiones de mayor volumen, las de mayor profundidad y aquellas localizadas en la punta nasal, labio y región parotídea, observándose como tejido fibroadiposo localizado o piel cicatricial con o sin telangectasias superficiales ${ }^{1,2}$.

Clínicamente se presentan como lesiones heterogéneas variando según la distribución, profundidad, localización y etapa evolutiva en que se encuentren ${ }^{1,3}$. Los HI pueden distribuirse de manera segmentaria, siguiendo dermatomos, o de manera focal. Según la pro- fundidad, pueden comprometer sólo la dermis (hemangiomas superficiales), sólo el tejido celular subcutáneo (hemangiomas profundos) o ambos (hemangiomas mixtos). Se ubican con mayor frecuencia en cabeza y cuello $(60 \%)$, seguido del tronco $(25 \%)$ y las extremidades $(15 \%)$, presentándose en un $80 \%$ de los casos como lesiones únicas ${ }^{1,3,4}$.

El 20\% de los casos presenta complicaciones, principalmente las lesiones segmentarias y los HI que se encuentran en fase de crecimiento, siendo las más frecuentes la ulceración y la compresión u obstrucción de estructuras vecinas, lo que varía según su localización ${ }^{1}$.

El diagnóstico de los $\mathrm{HI}$ es clínico. El estudio de imágenes se utiliza como complemento diagnóstico en los casos dudosos, en las lesiones hepáticas y en los casos de hemangiomatosis neonatal benigna para descartar compromiso visceral ${ }^{1}$. Los más utilizados son la ultrasonografía con doppler y la resonancia magnética (RM).

La mayoría de los HI, por su ubicación y tamaño, no requiere tratamiento específico dado su curso benigno y auto limitado. Sin embargo, un $10-15 \%$ de los pacientes deben ser tratados durante la fase proliferativa ${ }^{6,7}$ ya que durante esta fase pueden comprometer estructuras vitales (vía aérea), pueden alterar la función de ciertos órganos (perioculares, hepáticos), pueden ulcerarse (causando dolor importante y aumentando el riesgo de infecciones) y pueden producir alteraciones estéticas de importancia. Tradicionalmente los corticoides sistémicos en altas dosis y por períodos prolongados han 
sido el tratamiento de primera línea, con las consecuentes complicaciones derivadas de sus efectos adversos. Recientemente se ha reportado el uso de Propranolol para el tratamiento de los HI, observándose efectos terapéuticos notablemente superiores a los observados históricamente con corticoides orales, sugiriéndose como fármaco de primera línea dado su conocida seguridad avalada por años de uso en cardiología ${ }^{6,9,10,11}$.

El objetivo de este estudio es evaluar esta nueva terapéutica en un número limitado de pacientes chilenos con hemangiomas de la infancia con indicación de tratamiento sistémico.

\section{Material y Método}

Estudio descriptivo observacional realizado en la Unidad de Dermatología del Hospital Luis Calvo Mackenna.

Se analizaron las características clínicas de 30 pacientes con diagnóstico de HI que requerían tratamiento sistémico y que fueron tratados con Propranolol entre Julio de 2008 y Junio de 2010.

Los HI tratados con Propranolol fueron aquellos localizados en regiones con repercusión vital, funcional o cosmética, tales como vía aérea, región peri orbitaria y peri genital, los hemangiomas con distribución segmentaria y aquellos con complicaciones locales tales como ulceración, que en otra terapia debiese haber recibido corticoides orales en altas dosis.

Previo al inicio del tratamiento los pacientes fueron evaluados en la Unidad de Cardiología del Hospital Luis Calvo Mackenna, con examen físico y electrocardiograma, con el objetivo de identificar posibles contraindicaciones para el uso del medicamento. Por otra parte, se educó a los padres para reconocer y prevenir los posibles efectos adversos del tratamiento tales como hipotensión, bradicardia, obstrucción bronquial e hipoglicemia.

La dosis de Propranolol usada fue de $2 \mathrm{mg} /$ $\mathrm{kg} /$ día dividido en 2 tomas diarias. Se administró en forma de suspensión oral de $4 \mathrm{mg} /$ $\mathrm{ml}$, formulación preparada en la farmacia del hospital. Los pacientes fueron controlados a las 24 horas de iniciado el tratamiento, luego semanalmente por un mes y luego mensualmente hasta suspender el medicamento.

En cada control se evaluó clínicamente la lesión, se tomó presión arterial y frecuencia cardíaca y se dejó un registro iconográfico de la lesión, herramienta que permitió el monitoreo de los cambios de tamaño, color y signos de involución. Además se realizó ultrasonografía con doppler en algunos hemangiomas para caracterizar los cambios secundarios al tratamiento y en los casos de hemangiomatosis neonatal benigna para descartar compromiso visceral.

\section{Resultados}

Treinta pacientes fueron tratados con Propranolol (tabla 1). De estos, 23 (77\%) fueron de sexo femenino y $7(23 \%)$ fueron de sexo masculino.

Según el momento de aparición, en 14 pacientes $(47 \%)$ el HI se observó desde el nacimiento y en 16 pacientes (53\%) el hemangioma apareció en los primeros días o semanas de vida.

De los hemangiomas tratados 12 eran segmentarios $(40 \%)$ y 18 eran focales $(60 \%)$. Dieciseis eran hemangiomas superficiales (53\%), 3 eran profundos $(10 \%)$ y 11 eran mixtos $(37 \%)$. Veintisiete pacientes $(90 \%)$ presentaron hemangiomas únicos y 3 pacientes $(10 \%)$ presentaron hemangiomas múltiples.

En 20 pacientes $(67 \%)$ el hemangioma se ubicó en cabeza y cuello: 6 peri oculares (figura 1), 3 labiales, 2 nasales, 2 en mejilla, 1 en región parotídea comprometiendo el conducto auditivo externo (figura 2), 1 en cuero cabelludo y 1 laríngeo. Cuatro pacientes presentaron HI que comprometían más de un segmento de la cara (1 peri ocular y labio, 1 mejilla y labio y 2 naso labiales).

En 5 pacientes (17\%) el HI se ubicó en el tronco: 2 en la región genital, 2 en la región inguinal y 1 en el tórax.

Tres pacientes $(10 \%)$ presentaron un $\mathrm{HI}$ ubicado en las extremidades. Todos fueron segmentarios (figura 3 ).

Por otra parte, 2 pacientes $(6 \%)$ presentaron múltiples hemangiomas focales en distintos segmentos corporales. 
Tabla 1. Pacientes con HI tratados con Propranolol

\begin{tabular}{|c|c|c|c|c|c|c|c|c|c|c|}
\hline $\begin{array}{l}\text { Pa- } \\
\text { ciente }\end{array}$ & Sexo & $\begin{array}{l}\text { Distri- } \\
\text { bución }\end{array}$ & Tipo & Ubicación & $\begin{array}{l}\text { Ulcera- } \\
\text { ción }\end{array}$ & $\begin{array}{l}\text { Edad } \\
\text { inicio } \\
\text { (días) }\end{array}$ & $\begin{array}{l}\text { Duración } \\
\text { (días) }\end{array}$ & $\begin{array}{l}\text { Efectos } \\
\text { adversos }\end{array}$ & $\begin{array}{l}\text { Respuesta a } \\
\text { tratamiento }\end{array}$ & Rebote \\
\hline 1 & M & Foc & Mix & Cuero cabelludo & Sí & 77 & 226 & No & Sí & No \\
\hline 2 & M & Seg & Sup & $\begin{array}{l}\text { Peri ocular y labio } \\
\text { superior }\end{array}$ & No & 155 & 97 & No & Sí & No \\
\hline 3 & $\mathrm{~F}$ & Seg & Mix & Naso labial & No & 112 & 270 & Irritabilidad & Sí & Sí \\
\hline 4 & $\mathrm{~F}$ & Seg & Mix & Mejilla y labio & No & 91 & 215 & No & Sí & Sí \\
\hline 5 & $\mathrm{~F}$ & Foc & Sup & Peri ocular & No & 100 & 145 & Irritabilidad & Sí & No \\
\hline 6 & $\mathrm{~F}$ & Seg & Sup & Extremidad superior & No & 30 & 45 & Irritabilidad & Sí & No \\
\hline 7 & $\mathrm{~F}$ & Foc & Sup & Nariz & No & 153 & 240 & Irritabilidad & Sí & Sí \\
\hline 8 & $\mathrm{~F}$ & Foc & Mix & Múltiples & No & 140 & $461 *$ & No & Sí & No \\
\hline 9 & M & Foc & Mix & Peri ocular & No & 82 & $390^{*}$ & No & Sí & No \\
\hline 10 & $\mathrm{~F}$ & Foc & Sup & Peri ocular & No & 109 & 300 & Irritabilidad & Sí & No \\
\hline 11 & $\mathrm{~F}$ & Seg & Mix & Parotídeo & Sí & 242 & $313^{*}$ & No & Sí & No \\
\hline 12 & M & Seg & Sup & Naso labial & Sí & 60 & 210 & Irritabilidad & Sí & No \\
\hline 13 & $\mathrm{~F}$ & Seg & $P$ & Laringe & No & 41 & 352 & Irritabilidad & Sí & No \\
\hline 14 & $\mathrm{~F}$ & Foc & Sup & Peri ocular & No & 300 & $221 *$ & No & Sí & No \\
\hline 15 & $\mathrm{~F}$ & Foc & Sup & Múltiples & No & 95 & $111^{*}$ & Irritabilidad & Sí & No \\
\hline 16 & $\mathrm{~F}$ & Foc & Sup & Región genital & No & 80 & $77^{*}$ & No & Sí & No \\
\hline 17 & $\mathrm{~F}$ & Seg & Sup & $\begin{array}{l}\text { Pectoral y extremidad } \\
\text { superior }\end{array}$ & No & 57 & $235^{*}$ & Irritabilidad & Sí & No \\
\hline 18 & $\mathrm{~F}$ & Foc & Sup & Peri ocular & No & 168 & $228^{*}$ & No & Sí & No \\
\hline 19 & $\mathrm{~F}$ & Foc & Sup & Mejilla & Sí & 104 & $200^{*}$ & No & Sí & No \\
\hline 20 & $\mathrm{~F}$ & Seg & Sup & Mejilla & No & 344 & $128^{*}$ & Irritabilidad & Sí & No \\
\hline 21 & $\mathrm{~F}$ & Foc & Sup & Nariz & No & 65 & 10 & No & Sí & No \\
\hline 22 & $\mathrm{~F}$ & Foc & Sup & Labio & Sí & 106 & $103^{*}$ & Irritabilidad & Sí & No \\
\hline 23 & $\mathrm{~F}$ & Seg & Mix & Extremidad superior & No & 60 & 310 & No & Sí & No \\
\hline 24 & $F$ & Seg & Mix & Región inguinal & Sí & 30 & 320 & Irritabilidad & Sí & No \\
\hline 25 & $\mathrm{~F}$ & Foc & Mix & Labio & No & 30 & $210^{*}$ & No & Sí & Sí \\
\hline 26 & M & Foc & $P$ & Peri ocular & No & 330 & $60^{*}$ & Insomnio & Sí & No \\
\hline 27 & $\mathrm{~F}$ & Foc & Mix & Labio & No & 50 & 245 & No & Sí & No \\
\hline 28 & M & Foc & Sup & Región genital & No & 60 & 180 & No & Sí & No \\
\hline 29 & M & Seg & Mix & Región inguinal & Sí & 120 & $90^{*}$ & No & Sí & No \\
\hline 30 & $\mathrm{~F}$ & Foc & P & Tórax & No & 180 & 360 & No & Sí & No \\
\hline
\end{tabular}

F: femenino. M: masculino. Foc: focal. Seg: segmentario. Mix: mixto. Sup: superficial. P: profundo. ${ }^{*}$ mantiene tratamiento al 30/06/2010.

La edad promedio de inicio del tratamiento con Propranolol fue de 4 meses (119 días), con un rango de 1 a 11 meses. Diez pacientes (33\%) habían sido tratados previamente: 9 con prednisona oral y 1 con prednisona oral y cirugía precoz. Por otra parte 4 pacientes, estando en tratamiento con prednisona oral, iniciaron Propranolol.

En el control a las 24 horas de tratamiento con Propranolol se observó disminución del color y brillo del HI en 24 pacientes $(80 \%)$. Desde el control del séptimo día en adelante 


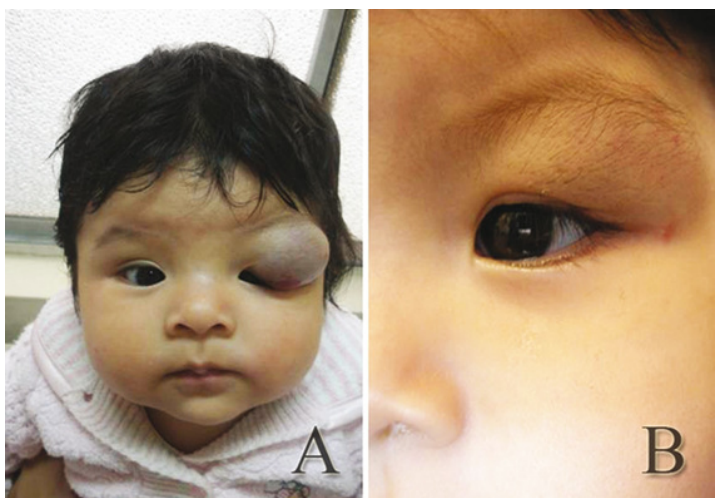

Figura 1. Paciente 8. Lactante portadora de hemangioma focal, mixto en el párpado superior izquierdo. A: al inicio del tratamiento, 4 meses de edad. B: al tercer mes de tratamiento, 7 meses de edad.

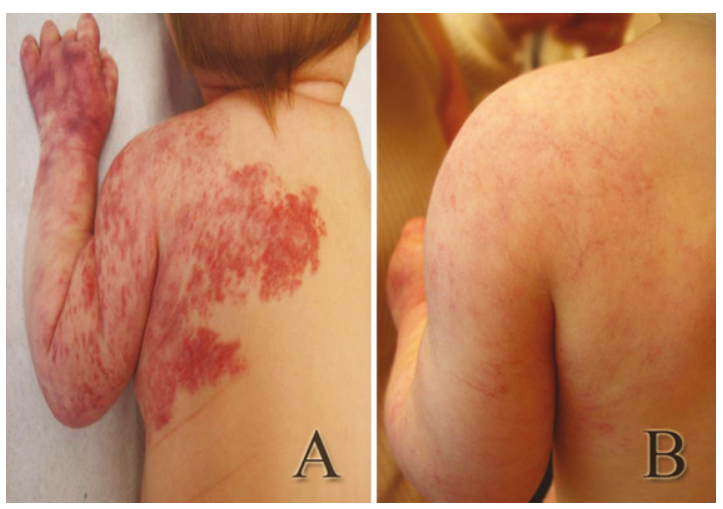

Figura 3. Paciente 17. Lactante portadora de hemangioma segmentario, superficial, que compromete el tronco y la extremidad superior izquierda completa. A: al inicio del tratamiento, 2 meses de edad. B: al séptimo mes de tratamiento, 9 meses de edad.

se observó en todos los pacientes, a través de la iconografía, que el uso de Propranolol frenó el crecimiento del HI durante el tratamiento y disminuyó la intensidad del color y el brillo. Además, aceleró el inicio de la involución en la totalidad de los pacientes.

En cuanto a los efectos adversos del tratamiento 13 pacientes $(43 \%)$ presentaron irritabilidad y trastornos del sueño. Ningún paciente presentó hipoglicemia, bradicardia, hipotensión, bronco espasmo ni disnea.

En la primera evaluación 7 pacientes $(23 \%)$ se presentaron con un HI ulcerado (figura 4). De estos, 4 pacientes habían sido tratados pre-

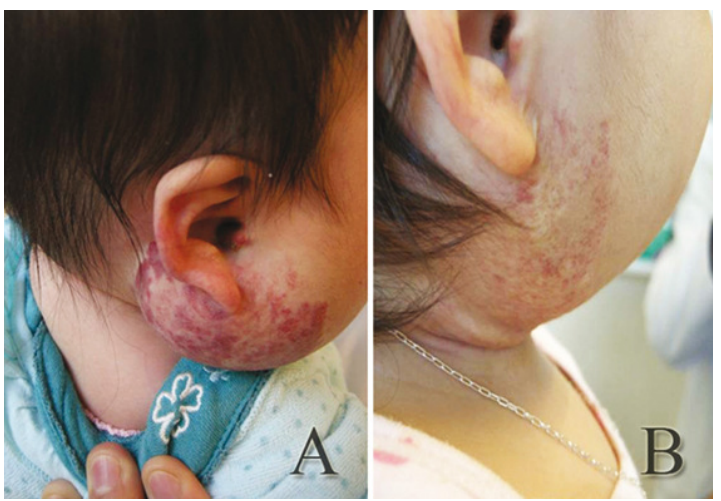

Figura 2. Paciente 11. Lactante portadora de hemangioma segmentario, mixto, ubicado en la región parotídea. A: al inicio del tratamiento, 8 meses de edad. B: al sexto mes de tratamiento, 1 año 2 meses de edad.
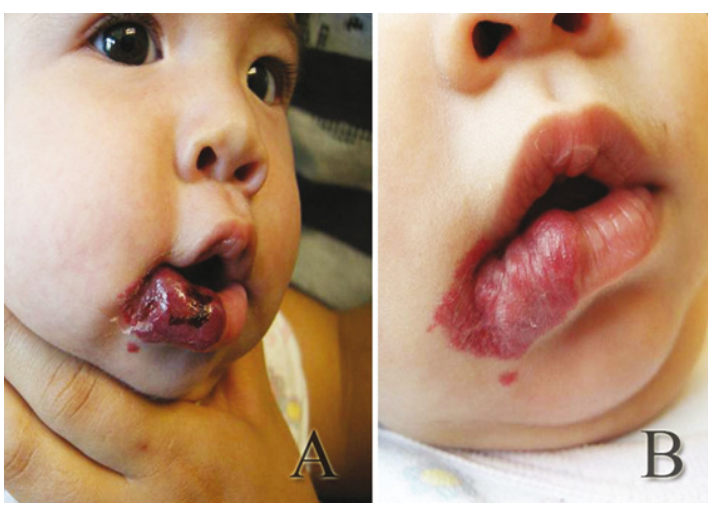

Figura 4. Paciente 22. Lactante portadora de hemangioma segmentario ulcerado del labio inferior. A: al inicio del tratamiento, 3 meses de edad. B: al tercer mes de tratamiento, 6 meses de edad.

viamente con prednisona oral, sin respuesta. Una vez iniciado el tratamiento con Propranolol la ulceración se resolvió en un promedio de 24 días (rango de 4 a 62 días). Ningún paciente presentó ulceración durante el tratamiento con Propranolol.

La duración promedio del tratamiento fue de 7 meses (212 días). Actualmente, 14 pacientes (47\%) mantienen tratamiento con Propranolol.

Luego de la suspensión del tratamiento 4 pacientes $(13 \%)$ presentaron rebote. En 2 de ellos se reinició el tratamiento con Propranolol observándose nuevamente una excelente respuesta. 


\section{Discusión}

La mayoría de los $\mathrm{HI}$ no requiere tratamiento específico, ya que tienen un curso benigno y auto limitado. Sin embargo, deben tratarse aquellos con distribución segmentaria, los que presentan complicaciones locales (por ejemplo ulceración), aquellos con alto riesgo de causar defectos cosméticos o funcionales importantes, tales como los ubicados en las regiones peri orificiales (orbitaria, auricular o genital), y aquellos con riesgo vital para el paciente, tales como los que comprometen la vía aérea y el hígado ${ }^{1,3,9,10,11}$.

Actualmente, los corticoides orales son la terapia de primera línea para el manejo de los $\mathrm{HI}^{1,3,6}$, pese a presentar una respuesta variable entre pacientes y a los múltiples efectos adversos derivados de su uso prolongado. La mayoría de estos efectos adversos son auto limitados, tales como la facie cushingoide, insomnio, irritabilidad, retardo en el crecimiento y síntomas gastrointestinales. Sin embargo, algunos pueden ser graves, tales como la hipertensión arterial y la miocardiopatía hipertrófica ${ }^{11}$. También se describe el uso de corticoides intralesionales, los que serían útiles en HI pequeños (menores de 2 a 3 centímetros de diámetro) y focales ${ }^{3}$.

Otras alternativas de tratamiento son la vincristina, el interferón alfa $2 \mathrm{~b}$ y la ciclofosfamida, pero su uso está restringido por sus importantes efectos adversos, tales como la toxicidad neurológica y hepática ${ }^{1,9,10,11}$.

La cirugía excisional tiene indicación en las secuelas cosméticas de HI involucionados, respetando las unidades estético funcionales de la zona a reparar. Por otra parte, la cirugía precoz es una herramienta útil en aquellas lesiones cuya localización puede amenazar la sobrevida del paciente o puede provocar un daño funcional importante, tales como los HI ubicados en el párpado, los que pueden producir ambliopía ${ }^{16-19}$.

El Propranolol es un fármaco antagonista no selectivo de los receptores beta adrenérgicos. Tiene una biodisponibilidad oral cercana al $26 \%$, se elimina por vía hepática casi en su totalidad y tiene una vida media de 4 a 5 horas $^{20}$. Su seguridad ha quedado demostrada en diversos reportes ${ }^{21-24}$, por lo que es ampliamente usado en pediatría para el manejo de diversas patologías tales como la hipertensión arterial, la taquicardia supra ventricular, el síndrome QT largo, la insuficiencia cardíaca, el hipertiroidismo y la prevención de crisis anoxémicas en la Tetralogía de Fallot ${ }^{9}$. Recientes estudios sugieren su uso para el manejo de los HI como una alternativa de tratamiento eficaz y segura, ya que actuaría no sólo frenando la fase de crecimiento del HI, sino que además disminuiría el tamaño tumoral en la fase proliferativa con mayor efectividad que los corticoides ${ }^{9}$.

Sus mecanismos de acción aún no son bien conocidos en HI. Sin embargo se postula que estimularía la vasoconstricción, induciría la apoptosis de las células endoteliales y disminuiría la angiogénesis de las células endoteliales modificando la expresión de los genes del factor de crecimiento vascular endotelial (VEGF) y del factor de crecimiento fibroblástico básico $(\mathrm{FGFb})^{1,6,9,10,31}$.

La dosificación habitual en pediatría es de $2-3 \mathrm{mg} / \mathrm{kg} /$ día y la dosificación propuesta en HI es de $1-3 \mathrm{mg} / \mathrm{kg} /$ día dividido en 2 a 3 tomas diarias. Cabe recordar que su intervalo de dosificación óptimo en general es cada 6 horas, pero se ha descrito mejor adherencia, sin modificar la respuesta, al administrarlo cada 8 a 12 horas $^{10}$. Todos nuestros pacientes fueron tratados con dosis de $2 \mathrm{mg} / \mathrm{kg} /$ día dividido en 2 tomas diarias, con excelente respuesta y adherencia al tratamiento, sin presentar efectos adversos de importancia. Su uso por décadas en niños, sin reportes de muerte ni secuelas incluso en intoxicaciones graves, le confiere al Propranolol un perfil de seguridad adecuado para nuestros pacientes con $\mathrm{HI}$.

Actualmente no existe consenso acerca de la duración del tratamiento con Propranolol, pero se sugiere que debiera cubrir toda la fase de crecimiento del HI con el objetivo de disminuir el riesgo de rebote.

Los principales efectos adversos del Propranolol descritos en la literatura son la hipoglicemia, hipotensión, bradicardia, irritabilidad, trastornos del sueño, bronco espasmo y disnea en pacientes con hiper reactividad bronquial, aunque estos efectos son en general dosis dependiente ${ }^{9,10}$. En esta casuística la mitad 
de los pacientes presentó cierta irritabilidad, especialmente al momento de dormir. No se presentaron eventos adversos importantes que provocaran la suspensión de la medicación.

Por otra parte, se ha reportado que el Propranolol tiene gran efectividad en el manejo de los hemangiomas de la vía aérea (tráquea, laringe) ${ }^{27-29}$. En nuestra serie, 1 paciente portador de un HI laríngeo hospitalizado en la UTI neonatal con estridor laríngeo y dificultad respiratoria presentó una dramática disminución de la signología obstructiva al tercer día de tratamiento con Propranolol, presentando además una mejoría evidente de la lesión en la visión endoscópica.

Recientemente se ha reportado el uso de Timolol tópico, un beta bloqueador no selectivo similar al Propranolol, como una alternativa efectiva y segura en los HI superficiales en etapa proliferativa ${ }^{31}$.

Los promisorios resultados observados con el uso de Propranolol en el tratamiento de los HI requieren protocolos de tratamiento para estandarizar su uso. Para ello, serán necesarios nuevos estudios clínicos controlados y prospectivos que permitan evaluar su eficacia, dosificación ideal y eventuales efectos adversos.

\section{Conclusión}

En nuestra serie el Propranolol fue una excelente herramienta terapéutica, eficaz y segura en el manejo de los hemangiomas de la infancia.

\section{Referencias}

1.- Drolet BA, Esterl N, Ilona J, Frieden I: Hemangiomas in Children. N Engl J Med 1999; 341: 173-81.

2.- Chang L, Haggstrom A, Drolet B, et al: Growth Characteristics of infantile hemangiomas: Implications for Management. Pediatrics 2008; 122: 360-7.

3.- Metry D, Hebert A: Benign cutaneous vascular tumors of infancy: when to worry, what to do. Arch Dermatol 2000; 136: 905-14.

4.- Kilcline C, Frieden IJ: Infantile Hemangiomas: how common are they? A systematic review of the medical literature. Pediatr Dermatol 2008; 25: 168-73.

5.- Hangstrom AN, Drolet BA, Beselga E, et al: Prospective study of infantile hemangioma: clinical characteristics, predicting complications and treatment. Pediatrics 2006; 118: 882-7.

6.- Léaute-Labréze C, Dumas de la Roque E, Hubiche T, Boralevi F, Thambo JB, Taieb A: Propranolol for severe hemamngiomas of infancy. N Engl J Med 2008; 358: 2649-51.

7.- Mulliken JB, Glowacki J: Hemangiomas and vascular malformations in infants and children: a classification based on endothelial characteristics. Plast Reconstr Sug 1982; 69: 412-20.

8.- Burrows PE, Laor T, Paltiel H, Robertson RL: Diagnostic Imaging in the evaluation of vascular birthmarks. Dermatol Clin 1998; 16: 455- 88.

9.- Frieden IJ, Drolet BA: Propranolol for infantile hemangioma: Promise, Peril, Pathogenesis. Pediatr Dermatol 2009; 26: 642-4.

10.- Lawley L, Siegfried E, Todd J: Propranolol treatment for hemangioma of infancy: risks and recommendations. Pediatr Dermatol 2009; 26: 610-4.

11.- Sanz V, Dumas de La Roque E, Berge J, et al: Propranolol for severe infantile hemangiomas: follow-up report. Pediatrics 2009; 124: 423-31.

12.- Bennett ML, Fleischer AB, et al: Oral corticosteroid use is effective for cutaneous hemangiomas. Arch Dermatol 2001; 137: 1208-13.

13.- Buckmiller LM: Propranolol treatment for infantile hemangiomas. Curr Opin Otolaringol Head Neck Surg. 2009; 17: 458-9.

14.- Hasan $Q$, Tan $S T$, et al: Effects of five commonly used glucocorticoids on hemangioma in vitro. Clin Exp Pharmacol Physiol 2003; 30: 140-4.

15.- Enjolras $O$, Riche MC, Merland J, Escande JP: Management of alarming hemangiomas in infancy: a review of 25 cases. Pediatrics. 1990; 85: 491-8.

16.- Morovic CG, Torres E, Florin C: Manejo quirúrgico precoz de Hemangiomas de Párpado. Rev Chil Cirug Plast. 2009; 7: 45-8.

17.- Morovic CG: Manejo Quirúrgico de los Hemangiomas Faciales en Niños. Rev Médica CLC 2009; 20: 829-34.

18.- Morovic CG, Vidal C, Acevedo C: Tratamiento quirúrgico de hemangiomas en niños. Rev Chil Pediatr 2000; 71: 17-23.

19.- Pitanguy I, Machado BH, Radwanski HN, Amorim NF: Surgical treatment of hemangiomas of the nose. Ann Plast Surg 1996; 36: 586-92.

20.- Zegpi MS, Nicklas C, Silva S: Actualización del uso de Invermectina Oral en niños y Propranolol en Hemangiomas de la Infancia. Rev Chil Derm 2010; 1: 5-8.

21.- Tan S, Itinteang T, Leadbitter P: Low-dose Propranolol 
KRAMER D. y cols.

for Infantile Hemangioma. Plast Reconstr Aesthet Surg 2010; 6: 40-2.

22.- Qin Z, Liu X, Li K, Yang X, Zheng J: Treatment for infantile hemangiomas with low-dose Propranolol: evaluation of short-term efficacy and safety. Zhonghua Yi Xue Za Zhi 2009; 89: 3130-4.

23.- Steven $N$, Weindling I, Philip S, Edward W: Efficacy and risks of medical therapy for supraventricular tachycardia in neonates and infants. Amer Heart Journal 1996; 131: 66-72.

24.- Artman M, Grayson M, Boerth R: Propranolol in children: safety-toxicity. Pediatrics 1982; 70: 30-31.

25.- Jeffrey N, Neal S: Are 1-2 Tablets Dangerous? BetaBlocker exposure in Toddlers. J Emerg Med 2004; 26: 309-14.

26.- Baetz J, Eigelshoven S, Marquard J, Bruch-Gerharz D, Homey B, Meissner T: Infantile hemangioma: successful treatment with Propranolol. Hautarzt 2010; 61: 290-2.

27.- Buckmiller L, Dyamenahalli U, Richter GT: Propranolol for airway hemangiomas: case report of novel treatment. Laryngoscope 2009; 119: 2051-4.

28.- Mistry N, Tzifa K: Use of Propranolol to treat multicentric airway hemangioma. J Laringol Otol 2010; 7: 1-4.

29.- Maturo $S$, Hartnick $C$ : Initial experience using Propranolol as the sole treatment for infantile airway hemangiomas. Int J Pediatr Otorhinolaryngol 2010; 74: 323-5.

30.- D'Angelo G, Lee H, Weiner RI: C AMP-dependent protein kinase inhibits the mitogenic action of vascular endotelial growth factor and fibroblast growth factor in capillary endotelial cells by blocking RAF activation. J Cell Biockem 1997; 67: 353-66.

31.- Pope E, Chakkittakandiyil A: Topical Timolol Gel for Infantile Hemangiomas: A Pilot Study. Arch Dermatol 2010; 146: 564-5. 